\title{
Minimizing opioid consumption following robotic surgery
}

\author{
Ruchika Talwar ${ }^{1,2}$, Shreyas S. Joshi ${ }^{3}$ \\ ${ }^{1}$ Division of Urology, Department of Surgery, Perelman School of Medicine, University of Pennsylvania, Philadelphia, PA, USA; ${ }^{2}$ Leonard Davis \\ Institute of Health Economics, University of Pennsylvania, Philadelphia, PA, USA; ${ }^{3}$ Division of Urologic Oncology, Department of Surgical \\ Oncology, Fox Chase Cancer Center, Philadelphia, PA, USA \\ Contributions: (I) Conception and design: All authors; (II) Administrative support: All authors; (III) Provision of study materials or patients: All \\ authors; (IV) Collection and assembly of data: All authors; (V) Data analysis and interpretation: All authors; (VI) Manuscript writing: All authors; (VII) \\ Final approval of manuscript: All authors. \\ Correspondence to: Shreyas S. Joshi, MD. Assistant Professor, Urologic Oncology, Fox Chase Cancer Center, Temple University Health System, 333 \\ Cottman Avenue, Philadelphia, PA 19111, USA. Email: shreyas.joshi@fccc.edu.
}

\begin{abstract}
The American opioid epidemic has led to one of the worse public health crises in recent history, and emerging evidence has highlighted the role of healthcare professionals in exposing patients and communities to potent opioid drugs. Surgeons, in treating postoperative pain, are at the forefront of this epidemic. In Urology, investigators are beginning to establish how patients handle and consume opioids following common urologic procedures in an effort to limit excess prescribing. However, there is a paucity of data to define acceptable amounts of opioid medications to adequately treat postoperative pain after urologic surgery. Many common urologic procedures are now routinely performed with robotic technology. Robotic, minimally-invasive approaches decrease incision size and accelerate postoperative recovery, thereby presenting a unique opportunity to curb excessive opioid prescribing in the postoperative patient. Herein, we explore the roots of the current crisis, outline current literature guiding pain control after surgery, and review the current, though sparse, literature that may guide urologists in decreasing opioid use after robotic surgery.
\end{abstract}

Keywords: Opioids; postoperative pain; robotic surgery

Submitted Apr 12, 2019. Accepted for publication Jul 30, 2019.

doi: $10.21037 /$ tau.2019.08.11

View this article at: http://dx.doi.org/10.21037/tau.2019.08.11

\section{A history of the American opioid epidemic}

Opioid derivatives have been utilized for centuries for their analgesic properties. During the era of the American Revolution, laudanum, an opioid based serum, was prescribed for pain control. Subsequent development of morphine led to an epidemic of addiction during the Civil War known as "Soldier's Disease" (1). By 1874, Bayer Pharmaceuticals advertised heroin as an analgesic with minimal addictive properties, leading to adoption of its use in medical practices (2). Unsurprisingly, opioid addiction became an undesirable consequence for many following heroin use, and legislators sought to address rising rates of addiction in the early 1900s by increasing taxes on opioid products and eventually banning heroin in the United
States (2). Although illicit heroin use persisted for years to come, the use of legal opioid substances skyrocketed by the turn of the 21 st century, creating unique and unprecedented challenges for the medical and public health communities to address rising addiction rates.

\section{Roots of a crisis: the roles of physicians, pharmaceutical companies, and government}

Although opioid misuse has recently gained nationwide attention, the roots of our current epidemic can be traced to a series of critical events that unfolded over the past 40 years implicating physicians, pharmaceutical companies, and federal agencies. The mainstreaming of scientific misinformation regarding opioids can be dated back 
to 1980. An approximately one hundred words, single paragraph "Letter to the Editor" published in the New England fournal of Medicine declared that addiction is rare in patients who are prescribed opioid medications based on a series of 11,882 inpatients, citing a less than $1 \%$ addiction rate (3). Despite offering virtually no insight regarding methodology and data analysis, this single article served as the basis of subsequent "evidence-based" claims supporting the safety profile of opioids with regards to addiction. A recent bibliometric analysis revealed that this index publication had been "uncritically cited" 608 times from its publication until March 2017, raising the concern that this extremely poor quality retrospective analysis served to inaccurately assuage provider fears regarding the risks of dependence from opioid medications (4).

Responding to the historical mismanagement of chronic pain in cancer and other conditions, in 1995 the American Pain Society introduced an initiative that trademarked pain as the "5th Vital Sign." In conjunction with the American Academy of Pain Medicine, the American Pain Society declared that there was insufficient evidence linking prescription opioid use with long-term addiction. By taking these positions, these and other organizations fostered an environment where providers were encouraged to utilize opioids as a humanistic approach to managing pathologic pain (5). A subsequent wave of support from the Veterans Affairs Medical System (VA), The Joint Commission (TJC), the American Medical Association (AMA), and various other professional and patient advocate groups led to congressional support for federal research funding on pain management (5).

The role of the pharmaceutical industry in fertilizing the opioid epidemic cannot be overstated. As the American Pain Society introduced the " 5 th Vital Sign" initiative, Purdue Pharmaceuticals (Stamford, CT, USA), the manufacturer of MSContin $^{\circledR}$ and OxyContin ${ }^{\circledR}$, became a substantial financial contributor to the organization (2). Upon release of OxyContin $^{\circledR}$ in 1995, Purdue Pharmaceuticals launched an elaborate advertising initiative and mobilized a large sales force to target nearly 100,000 physicians across the country who were deemed likely to prescribe the drug. They also utilized patient-centered promotions and coupons to offer a 30-day supply of drugs at no cost to the patient. Influenced by industry sponsored lectures and aggressive marketing campaigns, well-intentioned practitioners across the nation adopted liberal opioid prescribing practices (2).

Furthermore, in 1999 the VA also mandated the utilization of pain as the "5th Vital Sign," and in 2001 TJC included Pain Management Standards in their accreditation and reimbursement evaluation $(6,7)$. Pharmaceutical companies continued to co-sponsor the release of various guidelines on pain management that encouraged physicians to liberally prescribe opioid medications. They also invested millions of dollars in grants and support for research that minimized the addiction potential of prescription opioids, even in the absence of existing high quality evidence (8-10). Notably, this was a tactic borrowed from the tobacco industry, which conducted a decades-long "scientific" counter-messaging campaign in an attempt to create a body of literature to downplay the product's health dangers.

Pharmaceutical companies are now under increasing scrutiny for their culpability in promoting opioid overprescribing, and, in some cases, willfully deceptive product promotion. There is active litigation by federal, state, and individual plaintiffs against the pharmaceutical industry for their role in the opioid epidemic, with the outcomes still pending at the time of this publication.

\section{Opioid prescribing in the postoperative period}

Opioid medications are commonly utilized to address post-procedural pain in patients undergoing surgery. In an effort to curtail opioid prescribing in an era of rising opioid addiction rates, the Centers of Disease Control (CDC) created guidelines for opioid use in chronic disorders, but neglected to provide guidance regarding post-operative pain management (11). As a result, there is significant practice variability in postoperative pain management strategies for each type of operation, even within single institutions $(12,13)$. This is problematic for two specific reasons. First, unnecessary exposure to potentially addicting opioid medications should be avoided for all patients who do not actually require such medication. Second, overprescribing can lead to an excess of opioid pills in the homes of patients, which fosters potential for misuse, sale, or distribution by either the patient or close contacts who may have access to leftover pills.

Postoperative pain treatment serves as the initial exposure to opioids for many patients who undergo surgery. A landmark study by Brummett et al. analyzed an opioidnaive cohort of patients who underwent both major and minor surgical procedures using a nationwide insurance claims dataset, and demonstrated a $6 \%$ rate of persistent opioid use across all patients (14). Findings published in this study also challenged the notion that persistent use is caused by persistent postoperative pain. Despite the assumption 
that patients experience more pain after major surgery than minor surgery, the rates of persistent use were similar after both major and minor procedures (14). Interestingly, the most important risk factors independently associated with new, persistent use were the presence of preoperative pain disorders such as back pain, arthritis, and neurologic pain (14). This suggests that postoperative pain may not have been the catalyst for continued opioid use, and highlights the concern that surgery merely catalyzed exposure to opioids. In a similar large national dataset analysis, opioid-naive patients with cancer who underwent curative-intent surgeries demonstrated a $10 \%$ rate of persistent opioid use following surgery (15).

There is concerning evidence suggesting that even small numbers of opioid pills prescribed by a medical professional carry a significant risk of persistent use. Shah et al. analyzed 1.3 million opioid-naive patients who were discharged from the inpatient medical and post-surgical setting with an opioid prescription. They demonstrated an approximately $6 \%$ rate of persistent opioid use at 1 year, after only a single day's prescription. When prescribed greater than 7 days of opioids, the rate of persistent use jumped to $13.5 \%$ (16).

Though even minimal exposure to opioids may be detrimental to some patients, evidence suggests wide overprescribing of and massive overexposure to these drugs in the postoperative setting. Data from the general, gynecologic, thoracic, and orthopedic surgery literature show that approximately $70-80 \%$ of patients report having unused opioid pills following surgery $(13,17,18)$. These same studies demonstrated that the excess opioids are rarely discarded and are improperly stored $(13,17,18)$. Unused, leftover opioids serve as a danger not only to the patient, but also present an unchecked supply of opioids into the community. Such opioid diversion presents a significant challenge, as evidenced by data from The National Survey on Drug Use and Health (NSDUH) showing that 50\% of adults with opioid use disorders obtained opioids from family/friends at no cost. Furthermore, an additional $15 \%$ stated they were able to purchase leftover pills from family/ friends (19).

\section{Opioid use after urologic surgery}

Urologists are also responsible for opioid overprescribing in the postoperative setting. A study utilizing the Healthcare Cost and Utilization Project (HCUP) database estimated the incidence of either opioid dependence or overdose approached 1 in 1,000 patients who underwent urological surgery from 2007 to 2011 (20). In 2011, Bates et al. retrospectively administered surveys to a cohort of 587 patients from 2-4 weeks after urologic surgery, and demonstrated that $67 \%$ of patients reported having excess, unused opioids (21). Concerningly, $91 \%$ of those patients retained those pills at home for future use (21). A similar prospective analysis of a smaller cohort who underwent prostate or kidney surgery at a single institution revealed that $60 \%$ of all prescribed opioids remained unused (22). Again, of these patients with excess opioids, $60 \%$ retained them in an improper manner, raising the possibility of opioid diversion (22).

A study by Patel et al. collected prospective data in both the open and robotic radical prostatectomy population with respect to pain needs and opioid use upon discharge. Again, the majority of prescribed opioids, $77 \%$ overall, went unused (23). This study measured the exact amount of oral morphine equivalents (OMEQ) prescribed and consumed by patients postoperatively. Although a median of $225 \mathrm{mg}$ OMEQ were prescribed in the overall cohort, a median of only $22.5 \mathrm{mg}$ OMEQ, or $10 \%$ of prescribed OMEQ, were actually consumed. On multivariable analysis, larger OMEQ prescriptions at discharge were a significant predictor of increased opioid utilization (23). Furthermore, although no patients reported persistent opioid use 30 days after surgery, $87 \%$ retained unused pills. Of the $13 \%$ who disposed their leftover pills, only $9 \%$ did so in an appropriate manner (23).

In order to specifically address the issue of opioidkeeping after urologic procedures, Cabo et al. collected survey data from a single institution to elucidate patients' rationale behind narcotic retention. Forty-four percent of patients cited the fear of return of disease-specific pain as the primary motivation behind opioid keeping, while $29 \%$ reported concerns regarding unrelated pain. The authors administered a handout outlining FDA approved opioid disposal methods and measured rates of narcotic retention pre- and post- intervention, and observed no significant decrease in rates of retention ( $72 \%$ versus $68 \%$ ) (24).

As these studies demonstrate, urology patients face the same issues as other post-surgical patients with regard to opioid exposure, retention, and diversion. Therefore, it is imperative that clinicians incorporate standardized protocols to minimize opioid prescribing in this patient population.

\section{Reducing opioid consumption after robotic surgery}

The safety and occasional superiority of minimally invasive 
Opioid Free Post-Operative Pain Pathway

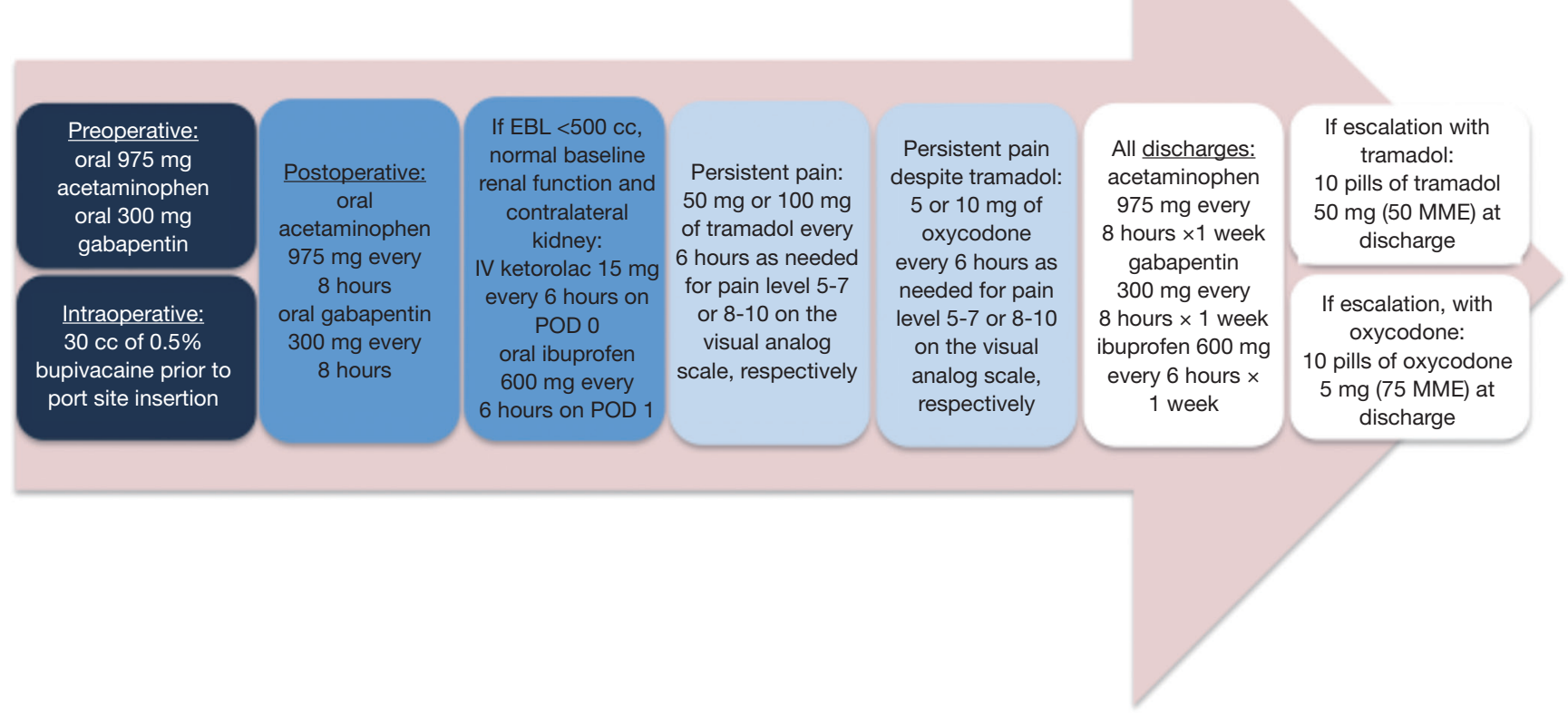

Figure 1 Multimodal non-opioid analgesic pathway. Adapted from Division of Urology, University of Pennsylvania.

approaches to common urologic procedures, such as radical prostatectomy, have led to the widespread adoption of robotic surgery within the field. It has been widely accepted that robotic surgical approaches result in less pain than open approaches $(25,26)$. However, evidence using validated measures of pain-related quality of life metrics comparing robotic and open surgeries do not exist. There is only one, single institutional, survey based observational study that demonstrated no difference between postoperative opioid consumption in robotic versus open prostatectomy (23), and no such data exists with respect to any other robotic urologic procedure. However, extrapolating from the literature, we propose a multi-tiered approach to effective opioid stewardship, including the use of multimodal analgesia and targeted local anesthetics, utilization of statelevel drug monitoring programs, and the use of standardized opioid prescribing guidelines.

Several pharmaceutical strategies have been shown to decrease opioid requirements after robotic urologic surgery. Multimodal analgesic strategies have been shown to significantly decrease opioid requirements upon discharge in patients undergoing penile prosthesis surgery (27). A study from this author's home institution implemented a standardized, multimodal non-opioid analgesic pathway consisting of preoperative gabapentin and acetaminophen, intraoperative local anesthetic infiltration prior to incision, and postoperative standing gabapentin, acetaminophen, and ketorolac (Figure 1). Escalation options were offered for patients with persistent pain not controlled without opioids. Tramadol was used as a first-line escalation agent, followed by oxycodone. Only patients who required escalation were discharged with ten pills of the corresponding opioid. This protocol (see Figure 1) may allow most patients undergoing robotic prostatectomy (RARP) and robotic radical (RARN) or partial nephrectomy (RAPN) to be discharged without any opioid prescriptions. Pre-implementation of this protocol, $100 \%$ of patients were discharged with varying amounts of $5 \mathrm{mg}$ oxycodone pills. Figure 2 illustrates postimplementation rates of discharge without opioids, with tramadol, and with oxycodone after robotic surgery (28).

In addition to the use of routine non-opioid analgesics, targeted local anesthetic agents should be prioritized as a way to improve pain control after robotic surgery. In a recent double-blind trial with robotic prostatectomy, patients were randomized to receive local anesthetic infiltration of the wound with or without ultrasound- 

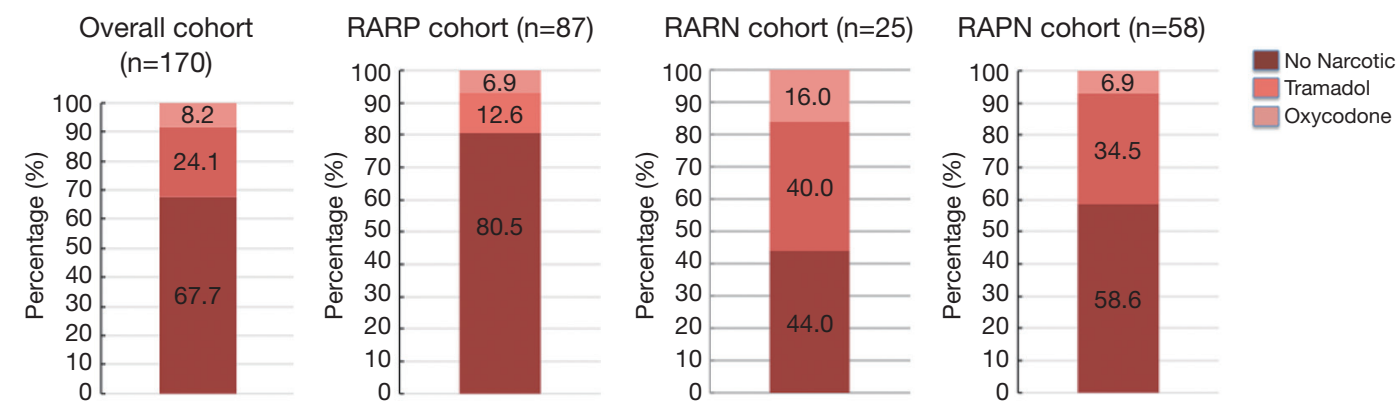

Figure 2 Unpublished data from author's institution: discharge prescription following adoption of the multimodal non-narcotic opioid analgesia pathway.

guided transversus abdominis plane (US-TAP) nerve block. The investigators demonstrated that patients in the US-TAP cohort had lower rates of opioid consumption following surgery. The authors also demonstrated a shorter length of stay and lower use of GI-motility agents in this cohort (29). Another study evaluated the efficacy of the TAP block when added to total intravenous anesthesia (TIVA) in patients undergoing robotic radical prostatectomy, and found that that the use of nerve blockade plus TIVA, as opposed to TIVA alone, resulted in lower amounts of opioid consumption during the perioperative period (30).

Prescription drug monitoring programs (PDMP) are another tool to decrease opioid over-prescribing. PDMPs have been independently implemented by every state in the United States. The goal of PDMPs is to track prescription, dispensation, and utilization records for all controlled substance prescriptions within each state. They serve as an important tool for clinicians to monitor patient prescriptions and avoid overprescribing. However, the effectiveness of such programs has not been clearly established. Some well-studied states, such as Florida, do appear to have seen changes as a result of PDMP implementation. For example, a comparative time series analysis between Florida and Georgia found that Florida's PDMP implementation resulted in a statistically significant decrease in outpatient opioid administration in the state, with the highest impact coming from those providers with higher baseline prescribing practices (31). Another study examining the Florida population demonstrated a $25 \%$ decrease in oxycodone-related mortality within only one month of PDMP implementation (32). However, not all studies appear to demonstrate overall PDMP effectiveness, as evidenced by an analysis of practices in New Hampshire evaluating 1,057 patients undergoing general surgical procedures. In this analysis investigators failed to find any significant decrease in opioid prescribing rates related to PDMP implementation (33).

Lastly, utilizing evidence-based, procedure-specific guidelines for opioid prescribing could help minimize variations in opioid prescription patterns. After finding that the majority of post-surgical patients were only using $38 \%$ of opioids prescribed upon discharge, Hill et al. developed a prescribing algorithm that would satisfy approximately $80 \%$ of outpatient opioid requirements for each procedure at their institution (34). Implementation of the algorithm yielded impressive results, as less than $1 \%$ of patients required an opioid refill. Furthermore, this intervention resulted in a $53 \%$ decrease in overall opioid prescribing (34). The Michigan Surgical Quality Collaborative recently developed similar guidelines based on prescribing recommendations designed to satisfy $75 \%$ of outpatient opioid requirements. The Collaborative has designed downloadable opioid prescribing guidelines, as well as provider and patient resources to guide perioperative counseling regarding pain control expectations and practice patterns (35). There have yet to be any similar guidelines published within urology, however. The American Urological Association released an AUA Position Statement on Opioid Use, but has not recommended specific prescription amounts or recommended opioid therapy duration following urologic procedures (36).

\section{Conclusions}

Healthcare professionals have played an important role in the United States opioid epidemic by overprescribing opioids, which have led many patients to develop chronic opioid dependence disorders. Overprescribing has also harmed non-patients by exposing entire communities to leftover, unused prescription opioids. Historically, our 
understanding of how to best treat postoperative pain was poor, and evidence about optimal pain management strategies was sparse. Poorly-conceived, though wellintentioned, standards for pain management in the past few decades led to widespread opioid overprescribing in an effort to never "undertreat" pain. With the growing appreciation of the opioid epidemic as a national public health emergency, it has become clear that there are significant gaps in medical literature regarding the biologic factors behind the experience of pain, the effective treatment of pain, and management of pain in patients with opioid dependence (37).

Because surgeons treat acute pain after surgery, they play a unique role in the healthcare industry's contribution to the opioid epidemic. Within urology, robotically-assisted laparoscopic surgery is extremely common; and smaller incisions and quicker postoperative recovery might afford an opportunity to reduce opioid overprescribing in this patient population. Limiting opioid use through the use of multimodal analgesia and targeted local anesthetic blocks has been proven to be a safe and effective way to manage postoperative pain following robotic urologic surgeries. Furthermore, the use of PDMPs and evidence-based prescribing guidelines also provides potential avenues of guidance for providers prescribing opioids after surgery.

Although randomized, controlled trials comparing non-opioid and opioid analgesia after robotic urologic surgery do not currently exist, it is clear that medical professionals, and urologists specifically, are overprescribing opioid medications after surgery. As evidence mounts demonstrating the utility of non-opioid treatment pathways, it is essential to continue to modify current institutionaland provider-level practice patterns to eliminate our contribution to the opioid epidemic.

\section{Acknowledgments}

Funding: None.

\section{Footnote}

Provenance and Peer Review: This article was commissioned by the Guest Editors (Marc C. Smaldone and Jeffrey J. Tomaszewski) for the series "Controversies in Minimally Invasive Urologic Oncology" published in Translational Andrology and Urology. The article has undergone external peer review.
Conflicts of Interest: Both authors have completed the ICMJE uniform disclosure form (available at http://dx.doi. org/10.21037/tau.2019.08.11). The series "Controversies in Minimally Invasive Urologic Oncology" was commissioned by the editorial office without any funding or sponsorship. The authors have no other conflicts of interest to declare.

Ethical Statement: The authors are accountable for all aspects of the work in ensuring that questions related to the accuracy or integrity of any part of the work are appropriately investigated and resolved.

Open Access Statement: This is an Open Access article distributed in accordance with the Creative Commons Attribution-NonCommercial-NoDerivs 4.0 International License (CC BY-NC-ND 4.0), which permits the noncommercial replication and distribution of the article with the strict proviso that no changes or edits are made and the original work is properly cited (including links to both the formal publication through the relevant DOI and the license). See: https://creativecommons.org/licenses/by-nc-nd/4.0/.

\section{References}

1. Hyams KC, Wignall FS, Roswell R. War syndromes and their evaluation: from the U.S. Civil War to the Persian Gulf War. Ann Intern Med 1996;125:398-405

2. deShazo RD, Johnson M, Eriator I, et al. Backstories on the US Opioid Epidemic. Good Intentions Gone Bad, an Industry Gone Rogue, and Watch Dogs Gone to Sleep. Am J Med 2018;131:595-601.

3. Porter J, Jick H. Addiction rare in patients treated with narcotics. N Engl J Med 1980;302:123.

4. Leung PTM, Macdonald EM, Stanbrook MB, et al. A 1980 letter on the risk of opioid addiction. N Engl J Med 2017;376:2194-5.

5. Brennan F. The US congressional "Decade on pain control and research" 2001-2011; a review. J Pain Palliat Care Pharmacother 2015;29:212-27.

6. Mularski RA, White-Chu F, Overbay D, et al. Measuring pain as the 5 th vital sign does not improve quality of pain management. J Gen Intern Med 2006;21:607-12.

7. Joint Commission for Accreditation of Health Organizations (JCAHO). Improving the Quality of Pain Management through Measurement and Action. Oakbrook Terrace, IL: Joint Commission Resources, Inc; 2003.

8. Joranson DE, Gibson A, Dahl JL, et al. Pain 
management, controlled substances, and state medical board policy: a decade of change. J Pain Symptom Manage 2002;23:138-47.

9. Federation of State Medical Boards. Model policy on the use of opioid analgesics in the treatment of chronic pain; 2017. Available online: https://www.fsmb.org/siteassets/ advocacy/policies/opioid_guidelines_as_adopted_ april-2017_final.pdf

10. Fauber J. UW a force in pain drug growth. Milw J Sentinel 2011;412. Available online: http://archive.jsonline.com/ watchdog/watchdogreports/119130114.html/. Accessed March 1, 2019.

11. Dowell D, Haegerich TM, Chou R. CDC guideline for prescribing opioids for chronic pain-United States, 2016. MMWR Recomm Rep 2016;65:1-49.

12. Paulozzi LJ, Hockenberry JM. Vital signs: variation among States in prescribing of opioid pain relievers and benzodiazepines-United States, 2012. MMWR Morb Mortal Wkly Rep 2014;63:563-8.

13. Hill MV, Stucke RS, Barth RJ. Wide variation and excessive doage of opioid prescriptions for common general surgical procedures. Ann Surg 2017;265:709-14.

14. Brummett CM, Waljee JF, Goesling J, et al. New persistent opioid use after minor and major surgical procedures in US adults. JAMA Surg 2017;152:e170504.

15. Lee, JS, Hu HM, Edelman AL, et al. New Persistent Opioid Use Among Patients With Cancer After CurativeIntent Surgery. J Clin Oncol 2017;35:4042-9.

16. Shah A, Marin BC. Characteristics of initial prescription episodes and likelihood of long-term opioid use - United States, 2006-2015. MMWR Morb Mortal Wkly Rep 2017;66:265-9.

17. Kim N, Abboudi J, Jones C, et al. A prospective evaluation of opioid utilization after upper-extremity surgical procedures: identifying consumption patterns and determining prescribing guidelines. J Bone Joint Surg Am 2016;98:e89.

18. Bartels K, Dingmann C, Bullard KJ, et al. Opioid use and storage patterns by patients after hospital discharge following surgery. PLoS ONE 2016;11:e0147972.

19. Lipari R. How people obtain the prescription pain relievers they misuse. The CBHSQ Report: January 12, 2017. Center for Behavioral Health Statistics and Quality, Substance Abuse and Mental Health Services Administration, Rockville, MD. Available online: https:// www.samhsa.gov/data/sites/default/files/report_2686/ ShortReport-2686.html. Accessed March 1, 2019.

20. Shah AS, Blackwell RH, Kuo PC, et al. Rates and Risk
Factors for Opioid Dependence and Overdose after Urological Surgery. J Urol 2017;198:1130-6.

21. Bates C, Laciak R, Southwick A, et al. Overprescription of postoperative narcotics: a look at postoperative pain medication delivery, consumption and disposal in urological practice. J Urol 2011;185:551-5.

22. Theisen KM, Myrga JM, Hale N, et al. Excessive Opioid Prescribing After Major Urologic Procedures. Urology 2019;123:101-7.

23. Patel HD, Srivastava A, Patel ND, et al. A Prospective Cohort Study of Postdischarge Opioid Practices After Radical Prostatectomy: The ORIOLES Initiative. Eur Urol 2019;75:215-8.

24. Cabo J, Hsi RS, Scarpato KR. Postoperative Opiate Use in Urological Patients: A Quality Improvement Study Aimed at Improving Opiate Disposal Practices. J Urol 2019;201:371-6.

25. Tewari A, Srivastava A, Menon M. Members of the VIP team. A Prospective Comparison of Radical Retropubic Prostatectomy and Robot-Assisted Prostatectomy: Experience in One Institution. BJU Int 2003;92:205-10.

26. Woldu SL, Weinberg AC, Bergman A, et al. Pain and analgesic use after robot-assisted radical prostatectomy. J Endourol 2014;28:544-8.

27. Tong CMC, Lucas J, Shah A, et al. Novel Multi-Modal Analgesia Protocol Significantly Decreases Opioid Requirements in Inflatable Penile Prosthesis Patients. J Sex Med 2018;15:1187-94.

28. Talwar R, Xia L, Serna J, et al. Preventing excess narcotic prescriptions in MIS urologic oncology discharges (PENN): A prospective cohort quality improvement initiative. J Clin Oncol 2019;37:6502.

29. Cacciamani GE, Menestrina N, Pirozzi M, et al. Impact of Combination of Local Anesthetic Wounds Infiltration and Ultrasound Transversus Abdominal Plane Block in Patients Undergoing Robot-Assisted Radical Prostatectomy: Perioperative Results of a Double-Blind Randomized Controlled Trial. J Endourol 2019;33:295-301.

30. Noguchi S, Saito J, Nakai K, et al. Efficacy of abdominal peripheral nerve block and caudal block during robotassisted laparoscopic surgery: a retrospective clinical study. J Anesth 2019;33:103-7.

31. Rutkow L, Chang HY, Daubresse M, et al. Effect of Florida's Prescription Drug Monitoring Program and Pill Mill Laws on Opioid Prescribing and Use. JAMA Intern Med 2015;175:1642-9.

32. Delcher C, Wang Y, Young HW, et al. Trends in Florida's Prescription Drug Monitoring Program registration and 
utilization: Implications for increasing voluntary use. J Opioid Manag 2017;13:283-9.

33. Stucke RS, Kelly JL, Mathis KA, et al. Association of the Use of a Mandatory Prescription Drug Monitoring Program With Prescribing Practices for Patients Undergoing Elective Surgery. JAMA Surg 2018;153:1105-10.

34. Hill MV, Stucke RS, Billmeier SE, et al. Guideline for Discharge Opioid Prescriptions after Inpatient General Surgical Procedures. J Am Coll Surg 2018;226:996-1003.

Cite this article as: Talwar R, Joshi SS. Minimizing opioid consumption following robotic surgery. Transl Androl Urol 2021;10(5):2289-2296. doi: 10.21037/tau.2019.08.11
35. Michigan OPEN. Opioid Prescribing Recommendations for Surgery. Avaialble online: https://opioidprescribing. info. Accessed March 1, 2019.

36. American Urological Association Board of Directors. AUA Position Statement: Opioid Use. Avaialble online: https:// www.auanet.org/guidelines/opioid-use. Revised January 2019. Accessed March 1, 2019.

37. Gostin LO, Hodge JG, Noe SA. Reframing the opioid epidemic as a national emergency. JAMA 2017;318:1539-40. 